\title{
Agencia(s) indígena(s) en políticas públicas participativas en Norpatagonia: políticas de comanejo y relevamiento territorial
}

\author{
Samanta Guiñazú \\ Universidad Nacional Río Negro, Bariloche, Argentina. \\ Email:guinazusamanta@gmail.com \\ Florencia Trentini \\ Universidad Nacional de Quilmes, Buenos Aires, Argentina \\ Email:flortrentini@gmail.com \\ Nadia Ameghino \\ Universidad Nacional de Moreno, Buenos Aires, Argentina. \\ Email:ameghino.nadia@gmail.com
}

\begin{abstract}
Resumen: El artículo analiza la implementación de políticas públicas participativas e indigenistas en Norpatagonia. Articulando el enfoque etnográfico de políticas de comanejo y relevamiento territorial con análisis de normativas y documentos de gestión, profundiza las nociones de empoderamiento, cooptación y participación vinculadas a una visión esencializada de "lo indígena" operante en estas políticas. Asimismo, profundiza en formas situadas de agencia indígena que disputan y cuestionan formas modeladas de participación sobre las que se sostienen estas políticas, evidenciando intersticios entre empoderamiento y cooptación.
\end{abstract}

Palabras Clave: Agencia, políticas públicas, participación

\section{Indigenousagency in participatorypublicpolicies in Norpatagonia: comanagementpolicies and territorial survey}

\begin{abstract}
The article analyzes the implementation of participatory and indigenous public policies in Norpatagonia. Articulating the ethnographic approach of co-management policies and territorial survey with analysis of regulations and management documents, it deepens the notions of empowerment, cooptation and participation linked to anessentialized vision of "the indigenous" prevailing in thesepolicies. Fur ther more, it deepens into situated forms of indigenous agency that dispute and question the modeled forms of participation on which these policies are based, evidencing interstices between empowerment and cooptation.
\end{abstract}

Keywords: Agency, public policies, participation

\section{Agência indígena em políticas públicas participativas em Norpatagonia: políticas de co-gestão e levantamento territorial}

Resumo: O artigo analisa a implementação de políticas públicas participativas e indigenistas em Norpatagonia. Articulando a abordagem etnográfica das políticas de co-gestão e levantamento territorial com análise de regulamentos e documentos de gestão, aprofunda as noções de empoderamento, cooptação e participação vinculados a uma visão essencializada dos “indígenas” que operam nessas políticas. Da mesma forma, aprofunda-se nas formas situadas de 
agenciamento indígena que disputam e questionam arenas modeladas de participação sobre as quaisessas políticas se sustentam, evidenciando interstícios entre o empoderamento e a cooptação.

Palavras-chave: Agencia, políticas públicas, territorialidades, participação.

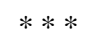

\section{Introducción}

En los últimos años, se vienen llevando adelante en Argentina políticas públicas participativas (PPP), implicando la participación de la sociedad en su diseño e implementación, e institucionalizando espacios de interacción entre estado y sociedad. En el caso de políticas indigenistas, esta participación está reconocida constitucionalmente y es resultado del accionar de la agencia indígena y de sus demandas específicas (Guiñazu, 2017). En este artículo analizamos dos casos etnográficos de PPP indigenistas implementadas en Argentina.Primero, comanejos de recursos y territorios establecidos por la Administración de Parques Nacionales (APN), focalizando en la relación entre los Parques Nacionales Lanín (PNL) (Ameghino, 2011, 2013) y Nahuel Huapi (PNNH) (Trentini, 2014, 2017) con comunidades del Pueblo Mapuche. Segundo, la implementación rionegrina del Relevamiento Territorial de Comunidades Indígenas (ReTeCI) ordenado por la Ley 26.160(Guiñazu, 2017, 2017b, 2018). Partiendo de este análisis, repensamos las nociones de empoderamiento, cooptación y participación fuertemente vinculadas a una visión esencializada de "lo indígena”. De este modo, daremos cuenta de formas situadas de agencia indígena que disputan y cuestionan las formas modeladas de participación sobre las que se sostienen este tipo de políticas, evidenciando "zonas grises”, e intersticios entre empoderamiento y cooptación.

En base a investigaciones previas entendemos que el desarrollo de estas políticas se basa en el supuesto de correspondencia entre "identidad cultural” y “territorio", moldeando un tipo de sujeto indígena "auténtico", sostenido mediante la acreditación y demostración de saberes, prácticas, y ocupación "ancestral” o "tradicional” de un espacio delimitado (Carenzo y Trentini, 2013). Esto parte de una visión esencialista que en la práctica condiciona la consecución de derechos y la misma participación que sustenta estas políticas. Frente a esto, los casos analizados exponen cómo los indígenas disputan, negocian y retoman este modelo para energizar la construcción de demandas políticas. En este sentido, entendemos a las PPP como espacios de contienda de múltiples intereses de los sujetos involucrados, de reconfiguración de límites y posibilidades de agencia y de disputa a las formas de juridicidad y politicidad que otorgan o niegan derechos. Estas formas no pueden entenderse sin atender alas subjetividades producidas mediante la incorporación contradictoria de los pueblos indígenas en el estado-nación-territorio argentino, y sin comprender el doble juego de exclusión/inclusión que ha marcado trayectorias y experiencias de comunidades indígenas a partir de particulares maneras de "producir alteridad” (Briones, 2005).

En este sentido, entendemos que la agencia indígena en el marco de PPP no debe pensarse a partir de la dicotomía dominación/resistencia (Ortner, 2016) o participación/cooptación, sino a partir de las prácticas cotidianas que dan cuenta de heterogeneidades, ambigüedades y contradicciones. Si estas políticas implican la posibilidad de "participar”, es necesario entender que esa participación debe pensarse en relación a las políticas de reconocimiento estatal y del modelo establecido por éstas. Por lo tanto, la(s) agencia(s) indígena(s) deben ser entendidas en función de las prefiguraciones establecidas por los dispositivos político-jurídicos que otorgan el reconocimiento y la posibilidad de participación. Así, deviene central considerar que la manera en que estas políticas modelan a "los indígenas" impacta sobre sus procesos de construcción identitaria, y que esta performatividad está relacionada a las orientaciones explícitas e implícitas establecidas en su definición e implementación y en relación a las formas de juridicidad, mediante las que el estado legaliza (o no) a estos grupos.

\section{Agencia indígena en políticas públicas participativas}

Las PPP se fundamentan en la participación y el involucramiento de los sujetos. Esta participación debe entenderse a partir de procesos organizativos y demandas que en Latinoamérica acompañaron la crisis del 
modelo neoliberal, encabezando procesos de movilización y participación política. Frente a esto, diferentes agencias gubernamentales e internacionales de financiación, promovieron instancias de "participación ciudadana". Entonces, las PPP implican una voluntad y apertura del estado hacia la participación de actores de la sociedad en sus procesos de construcción y ejecución. Esto es consecuencia de decisiones políticas orientadas a la adopción de un modo de gestión participativo, ya que el estado no está obligado a ser participativo. No obstante, en Argentina, la participación indígena está garantizada constitucionalmente y debe practicarse obligatoriamente en los casos de políticas que implican a pueblos indígenas (Guiñazú, 2017).

En este marco, analizamos la agencia indígena en el marco de PPP alejándonos de las dicotomías dominación/resistencia (Ortner, 2016) o participación/cooptación, asociándola a las formas situadas en que ésta se expresa. Así, daremos cuenta de la capacidad y heterogeneidad de esta agencia, de formas cotidianas de resistencia, respuesta e impugnación, de disputas de poder y representatividad, y de divergencias entre agendas, objetivos y prioridades intervinientes en la implementación de PPP. Asimismo, entendemos que las distintas expresiones de agencia tensionan las prefiguraciones y por ende el alcance de la performatividad de las políticas y de los sujetos implicados en las mismas.

En este sentido, entendemos que la agencia "nunca es un objeto en sí, sino que siempre forma parte de un proceso de (...) estructuración, es decir, la configuración y reconfiguración de formaciones sociales y culturales más amplias” (Ortner 2016, p.156). Profundizaremos esta noción de agencia recuperando tres elementos propuestos por Ortner (2016). Primero, la intencionalidad activa que distingue la agencia de prácticas rutinarias, no existiendo línea divisoria entre ambas, sino continuidad entre prácticas rutinarias llevadas a cabo con poca reflexión y planificación, y actos agentivos. Segundo, la simultaneidad del carácter universal y de construcción cultural de la agencia, remarcando que ésta se forma, nutre o debilita diferencialmente en distintos regímenes de poder. Tercero, la agencia enmarcada dentro de relaciones de poder y desigualdad, es siempre compleja y contradictoria, siendo la oposición sólo una de sus múltiples formas posibles. Así, la autora desarrolla que el poder consiste en destruir la agencia en tanto capacidad de llevar adelante proyectos activamente. De este modo, la agencia es una forma de propiedad de los sujetos sociales, formada culturalmente por medio de características resaltadas como agentivas. Se distribuye de modo desigual, y los sujetos pueden o no tener agencia, o pueden "tener más o menos" agencia. Sea cual fuera la agencia que parezcan "tener" como individuos, ésta siempre es negociada interactivamente, ya que nunca son agentes libres. Al respecto, Grossberg (2014) introduce la noción de movilidades estructuradas, producidas en el interjuego estratégico entre líneas de articulación (territorialización) y de trayectoria/fuga (desterritorialización). Mientras la diferencia entre esas líneas no corresponde a diferencias entre lugares y espacios, su naturaleza dinámica en la vida diaria es resultado es ese interjuego. Esto implica que no existe circulación alguna completamente libre y por fuera del poder, siempre estamos moviéndonos en espacios estructurados. Estas conceptualizaciones dan cuenta de un interjuego, de un ir y venir (Guiñazu, 2018), de una movilidad permanente que, lejos de establecer sólo fijezas, establece lugares para la acción, creatividad, construcción, reelaboración y actualización cotidiana a través de las prácticas de las personas.

Para este análisis adherimos a que las posiciones de los sujetos son resultado de los constreñimientos de las estructuras hegemónicas, así como de los lugares (físicos y/o sociales) habilitados para actuar en tanto agentes. Las expresiones de agencia indígena en contextos de reconocimiento estatal, se relacionan a este interjuego entre partes, donde se disputan, reelaboran, crean y actualizan límites y formas concretas para esa relación. De este modo, las agencias indígenas han adquirido, imaginado y expresado diversas formas, por medio de las cuales los actores hacen oír su voz, participan de diferentes performances, habilitan autoadscripciones, toman conciencia de trayectorias personales/colectivas, construyen e intensifican redes y alianzas, forman grupos de pertenencia de diversa trascendencia, etc.

Al mismo tiempo, enfatizamos la transformación del estado desde su interior por quienes ocupan sus espacios. Como mostraremos a lo largo del presente trabajo, el hecho de que algunos indígenas participen como técnicos o cumplan roles centrales "dentro" del estado han moldeado el hacer de la política, disputando sus tiempos, sus categorías y sus lineamientos. Así por ejemplo, el caso del relevamiento territorial muestra una clara disputa por lo que se entiende por "territorio tradicional" y el caso de comanejo da cuenta de la disputa por la propia categoría de "indígena”. En esa transformación, se flexibilizan y moldean demarcaciones y estructuras 
para incorporar a las diversas modalidades de agencia. Es decir, que el estado no es estático ni monolítico, y en sus prácticas y discursos presenta incoherencias, contradicciones y ambigüedades que le permiten reconstruirse, mutarse, y adaptarse para continuar encauzando posibles agencias emergentes.

Este enfoque permite analizar las PPP desde la tensión entre su definición y su construcción cotidiana, mostrando los múltiples modos en que se practican y definen, deviniendo espacios de disputa en el que interaccionan intereses, incluso contradictorios. Entonces, las políticas definen límites y posibilidades, que pueden ser desafiados y reconfigurados por quienes participan. Como sostienen Ramos y Delrio, las correspondencias naturalizadas entre determinados lugares y las construcciones estereotipadas de sus ocupantes son el punto de partida para comprender cuándo los sujetos indígenas devienen agentes capaces de modificar lo establecido (2005, p.79). En este sentido, es central entender cómo estas políticas performan, habilitan o prescriben determinadas formas de "ser y hacer indígena”, y cómo frente a ellas los sujetos despliegan diversas agencias.

\section{Agencia, performatividades y prescripciones}

Asociada la agencia a la idea de performatividad de las políticas públicas, en este caso participativas e indigenistas, seguimos a Briones (2007), para entender que el concepto de performatividad alude a encuadres de interpretación que encauzan la significación del discurso y de los actos, no sólo desde la perspectiva de los marcos conceptuales y pactos interaccionales, sino también de condicionamientos institucionales del comportamiento y producción del conocimiento (Briones, 2007). Esta performatividad refiere a beneficiarios y ejecutores de estas políticas, ya que las formas en que se expresan y ordenan construyen cómo los actores intervinientes son interpelados y se disponen a desarrollar el trabajo previsto, tensionando las formas de ejecución.

Así, acordamos con Briones (2007) respecto de que no es casual que el auge de los estudios críticos sobre la identidad ocurra en épocas signadas por "la politización de las identidades". El crecimiento y funcionamiento de este tipo de políticas ocurre en el devenir y articulación de voluntades políticas estatales con las tensiones y disputas de parte de la agencia indígena. Siguiendo su razonamiento, sostenemos que no es casual que estas PPP, emergidas en un contexto de mayor conflictividad territorial, adopten discursos, formas de prescripción, regulación y normalización, ocultas bajo un manto de inclusión, participación, interacción, democratización y horizontalidad (Guiñazu, 2017). Lo anterior puede entenderse como una lectura hegemónica de los reclamos indígenas, estatalmente traducidos a sus propios objetivos políticos, expresados en las formas mencionadas y complementadas con un análisis exhaustivo de quiénes son legítimos interlocutores para políticas como las que aquí analizamos.

Los casos propuestos dan cuenta de diversas formas de agencia indígena en PPP de comanejo y relevamiento territorial. A través de ellos mostraremos cómo el proceso de "encajar" en lo que estas políticas definen es un proceso de negociación y reconfiguración de los modelos establecidos, dando lugar a órdenes de juridicidad y politicidad ambiguos, que en ocasiones se encuentran a medio camino entre lo formal/legal y lo informal/ilegal, permitiendo disputar las formas de reconocimiento estatal (Trentini, 2017). Otros trabajos han profundizado en los modos en los que diferentes actores locales utilizan los espacios participativos de diferentes políticas públicas como espacios para crear redes y acceder a recursos que les permitan lograr mayor influencia en la política misma (Abers, 2007; Abers y Keck, 2009). No obstante, manifiestan que el escenario más común respecto de la participación en política pública da cuenta de que la misma tiene un impacto muy bajo en la toma de decisiones públicas, y que se trata de espacios adoptados formalmente pero que no implican espacios activos de debate. A diferencia de estos antecedentes, nuestro artículo recupera dos casos de PPP en los que la participación indígena ha tensionado y moldeado el curso previsto de las mismas.

\section{Los casos de análisis: comanejos y relevamiento territorial}

Focalizaremos en experiencias de comanejo en elPNL y PNNH y en la ejecución rionegrina del ReTeCI. Estos casos dan cuenta de diferentes relacionamientos entre estado e indígenas, mostrando cómo si bien las "fronteras" entre uno y otro son necesarias, también son flexibles y porosas. Partiendo del análisis etnográfico de sus prácticas mostraremos que estas "fronteras" se resignifican y modifican, evidenciando formas diferenciales de trabajo con y en el estado y mostrando particulares estrategias desplegadas en cada contexto de ejecución. 


\section{Comanejo de recursos y territorios}

A nivel de las arenas globales de conservación el comanejo es una alianza o acuerdo en el que dos o más actores negocian compartir responsabilidades de administración y manejo sobre un territorio o conjunto de recursos (Notzke, 1994). Estos arreglos varían el nivel de responsabilidad compartida y son percibidos como un modo de integrar al conocimiento científico occidental con el saber de comunidades locales o indígenas, para desarrollar prácticas sustentables.

Las políticas de comanejo son aplicadas como una solución puntual a conflictos irresueltos entre indígenas y estados. Desde esta concepción, los expertos en materia de conservación entienden la participación de pobladores locales como garantía de preservación de áreas protegidas a nivel mundial. Estos lineamientos son implementados en casos particulares en los que su definición no se discute, implicando un proceso lineal iniciado con una declaración de principios y convertido en legislación, aplicada a casos específicos. Nuestro análisis discute con esta visión, mostrando complejidad y desorden en los procesos de formulación e implementación de políticas de comanejo (Shore, 2010), revelando las maneras ambiguas y disputadas en las que estas políticas son vividas y experimentadas.

Para entender las políticas de comanejo en el marco de la APN, es necesario recordar que en arenas globales los parques nacionales han sido concebidos como espacios sin habitantes, pensados para resguardar los paisajes -supuestamente- naturales. Para los indígenas que habitan estos territorios, la constitución de estas áreas protegidas implicó violentas políticas de expulsión e invisibilización, violación y negación de derechos (Steven, 1997). Sin embargo, las últimas décadas manifiestan cambios en la concepción y gestión de estas áreas, entendiendo que el aislamiento y la intangibilidad no son la solución y que los derechos de estos pueblos y su participación deben ser asegurados (Borrini, 1997). Este cambio de paradigma dio lugar a la implementación de políticas de comanejo o manejos participativos.

A partir de 1970, estas modificaciones en la concepción de la conservación a nivel mundial focalizan en la participación de estas poblaciones en la gestión de las áreas protegidas (Amend y Amend, 1991). En Argentina, esta nueva relación se corporizó en políticas institucionales de la APN, a fines de 1990, en el comanejo del PNL, primera experiencia de estas características. En este contexto, los indígenas fueron lentamente incorporados en las políticas y acciones de conservación (Carpinetti, 2005). En este sentido, lo acontecido en el PNL es considerado como una "experiencia bisagra" en la relación de los pueblos indígenas con la APN, que posteriormente intentó replicarse en el PNNH.

Estos casos de comanejo deben entenderse en el marco de una política global que reconoce y revaloriza la importancia de comunidades indígenas, integrando aspectos culturales, sociales, económicos y políticos a un terreno previamente considerado como estrictamente ecológico y ecosistémico, consolidando a nivel global lo que Dumoulin (2005) define como "programa de la doble conservación”. Así, las experiencias argentinas de comanejo recuperan localmente cuestiones del ambientalismo global, principalmente la idea del "indio verde", entendiendo que los indígenas son socios privilegiados para conservar la naturaleza.

Desde 2004 existe una Resolución del Directorio de la APN que garantiza, a través del comanejo, la participación de las comunidades indígenas en todo acto administrativo, referido a recursos naturales en sus territorios. Además, el Plan de Gestión de la APN contempla el comanejo como "un reparto de responsabilidades y competencias, y una clara definición del ejercicio de la autoridad pública, y de las pautas de uso y manejo de los recursos que surgen del saber ancestral de las comunidades” (APN, 2001). Sin embargo, si bien en la mayoría de los parques nacionales habitan pueblos indígenas (o en áreas aledañas, en algunos casos por desalojos para la conformación de estas áreas), lo cierto es que no se están realizando estas experiencias más que en el PNL y en el PNNH con el Pueblo Mapuche.

El fortalecimiento institucional de la participación indígena en la APN se dio en un contexto que a priori parecía adverso: el proceso de reforma del estado, a partir de la puesta en marcha de un programa neoliberal. Se propuso reconfigurar el marco legal y normativo relativo al ambiente, bajo el discurso de la "modernización” y la incorporación de nuestro país en "el mundo". A esto se sumaba el creciente protagonismo de las ONGs 
ambientalistas involucradas en el rediseño del marco institucional de nuestro país. Así, aquellos criterios que habían guiado una política expulsiva y restrictiva, comenzaron a ser revisados y actualizados, ante la relevancia cobrada por "casos exitosos" de experiencias de conservación participativa. Estos cambios, además, deben entenderse en relación con el proceso de fortalecimiento y consolidación de las organizaciones de pueblos indígenas. Este movimiento hacia la apertura y participación de las poblaciones locales dentro de la APN fue recuperado por el personal técnico de terreno, quienes abrieron espacios participativos en colaboración en estas poblaciones (Carenzo y Trentini, 2013).

Hacia finales de 1999, la APN estaba sumida en una crisis política. Su personal técnico y administrativo luchaba contra el despido de técnicos, tercerización de servicios, y centralización de decisiones de manejo de áreas protegidas. En este marco, se desató un conflicto en la relación con las comunidades mapuche del PNL que culminó con la ocupación de la Intendencia del Parque por la Confederación Mapuche de Neuquén, proponiendo a la Administración realizar un taller para facilitar y fortalecer la relación y discutir territorialidad, legislación y manejo de recursos naturales (Carpinetti, 2005, p.51). El taller de “Territorio Indígena Protegido”, realizado en mayo del 2000, marcó un punto de inflexión en la relación entre comunidades y la APN, elaborando un nuevo marco conceptual y legal plasmado en el proyecto de comanejo del PNL. Todo esto evidenció un cambio concreto en las prácticas de la APN, regidas hasta ese momento por una lógica restrictiva y excluyente.

Cabe destacar el fortalecimiento de la lucha de las organizaciones indígenas en relación a las áreas protegidas, tanto en las demandas de reparación histórica por la expulsión de los territorios que hoy son áreas protegidas, como por su integración prioritaria en la gestión actual de estos espacios. El comanejo fue una demanda del Pueblo Mapuche y no dependió simplemente de la voluntad política de la APN, y esto debe pensarse en relación a la participación que referentes mapuche tienen en instancias internacionales (Congresos Mundiales de Parques Nacionales, Asambleas de la Unión Internacional de Conservación de la Naturaleza UICN) donde se definen lineamientos globales sobre esta política, partiendo de sus experiencias concretas. En este sentido, enfatizamos que la implementación de esta política no depende simplemente de una definición institucional, sino de procesos específicos en los que deviene fundamental la agencia de aquellos actores involucrados. Esto debe pensarse en relación al hecho de que más allá de las resoluciones institucionales no se han implementado comanejos en la mayoría de los parques nacionales de Argentina. Es decir que esta política no puede ser entendida simplemente desde el cambio de discurso político de la institución, en el marco de un cambio mundial de paradigma de la conservación, sino también de la agencia del Pueblo Mapuche en los casos de los parques Lanín y Nahuel Huapi.

\section{Relevamiento Territorial de Comunidades Indígenas}

El segundo caso de PPP a analizar es el relevamiento territorial de comunidades indígenas, enmarcado en la ley de Emergencia Territorial No 26.160 de 2006. Su principal objetivo fue la declaración de Emergencia de los territorios ocupados tradicionalmente por pueblos indígenas y la suspensión de desalojos. Estas dos acciones buscaban establecer un freno ante atropellos, desalojos y hechos violentos sufridos por comunidades en todo el país. Adicionalmente, se estableció la necesidad de conocer la realidad socio/territorial de estas comunidades, ordenando para ello un relevamiento de alcance nacional con presupuesto para llevarlo a cabo. De este modo, se delineó esta política que en su letra establecía sólo algunos pocos lineamientos, utilizando nociones ambiguas o indefinidas, generando discusiones sobre cómo implementar el relevamiento, sus limitaciones y alcances.

Para su ejecución, el Consejo de Participación Indígena (CPI) y el INAI dieron forma al ReTeCI. Diferentes versiones del programa se pusieron a consideración de representantes indígenas, y, finalmente, en marzo de 2007, se aprobó la versión final en el marco del Segundo Encuentro Nacional del CPI, luego de jornadas de trabajo en las cuales se expusieron, evaluaron y debatieron procedimientos técnicos y metodologías para su implementación. En este marco deco-construcción entre estado, representantes indígenas y comunidades, se forjó la forma final que tomó el relevamiento. Desde el INAI se estableció y plasmó en el Manual del relevamiento que uno de sus principales intereses era garantizar el reconocimiento de la posesión y propiedad comunitaria que tradicionalmente ocupan las comunidades indígenas. Además, se manifestó el interés en la promoción de la participación indígena en la elaboración, ejecución y seguimiento de los proyectos que deriven del programa. 
Así, el objetivo central del ReTeCI implicó el "relevamiento técnico, jurídico y catastral de la situación dominial de las tierras ocupadas por las comunidades indígenas”. De acuerdo a la Ley, las tierras a relevar, debían cumplir con el requisito de estar ocupadas de forma "actual, tradicional y pública".

Atendiendo a un objetivo de descentralización, se previó la conformación de Unidades Ejecutoras Provinciales (UP), integradas por representantes del ejecutivo provincial, de comunidades, y un equipo técnico operativo (ETO).Cada provincia tenía las facultades para iniciar el relevamiento de manera independiente, generando contrastes e inconvenientes en relación a su inicio. Acercándose la finalización de vigencia de la ley, muchas provincias no habían presentado ningún tipo de resultado de ejecución, por lo cual se dispuso prorrogarla. Esta dinámica diferencial entre provincias se debió a sus dispares contextos políticos, sociales y económicos. En algunos casos, las autoridades provinciales se negaron a iniciar el diálogo con el INAI para establecer modalidades de ejecución, manifestando desinterés y descompromiso por atender a la cuestión indígena. Las provincias que negaron la ejecución del ReTeCI son mayormente aquellas con una alta conflictividad con las comunidades. Por este motivo, muchas de estas comunidades se movilizaron, exigiendo e ideando alternativas para dar cumplimiento al ReTeCI.

Los fondos asignados para el relevamiento fueron destinados a las diferentes UP. La UP rionegrina fue el Consejo de Desarrollo de Comunidades Indígenas (CODECI), un organismo de co-gobierno con reconocimiento provincial, encargado de seleccionar a su ETO, formado por profesionales de diferentes disciplinas; de comunicar a las comunidades la vigencia del relevamiento, límites, alcances, beneficios y posibilidades, para atender a la consulta libre, previa e informada en torno a la aceptación del programa; y de planificar formas, metodologías y tiempos de procedimiento.

Respecto de la participación indígena, el INAI expuso en sus fundamentos, programas y líneas de trabajo que su principal propósito era asegurar el ejercicio pleno de los derechos de las comunidades y pueblos indígenas, garantizando el cumplimiento de lo establecido constitucionalmente. Además, expuso que entre sus acciones permanentes está el impulso de la participación de las comunidades en el diseño y gestión de "las políticas de Estado que las involucran, respetando sus formas de organización tradicionales”.

\section{Agencia(s) indígena(s) en la construcción cotidiana de políticas públicas participativas}

Las experiencias presentadas permiten pensar cuestiones vinculadas a las PPP e indigenistas que disputan su hacer cotidiano, sus definiciones y modelos. Es justamente dar cuenta de las relaciones entre las construcciones estereotipadas y la delimitación de determinados lugares habilitados para "los indígenas" las que permiten mostrar cómo estos devienen agentes capaces de modificar lo establecido (Ramos y Delrio, 2005). En este sentido, algunas cuestiones permiten pensar la política más allá de su definición, abordándola a partir de múltiples modos en que se practican y definen en su construcción cotidiana.

Desde este enfoque discutimos con la visión lineal de la formulación e implementación de los comanejos y el ReTeCI, mostrando cómo ambas políticas son arenas de disputa que se construyen y reconstruyen en su aplicación, desafiando y reconfigurando límites establecidos. También, repensamos las nociones de participación, cooptación y empoderamiento vinculadas a las mismas. Para esto ponemos en discusión el momento de inicio de estas políticas, la idea de "sujetos destinatarios" que las sostienen y la relación entre estado y sociedad civil que las sustentan.

\section{Agencia indígena en el armado de la política}

Las fechas de institucionalización en que ambas políticas iniciaron formalmente difieren de lo que llamaremos su "inicio real". Así, explicitaremos que se trata de PPP que implicaron una apertura a la participación desde el proceso de formulación y diseño. Es decir que la agencia y participación indígena fueron relevantes primero, para situar la problemática indígena en general y la territorial en particular como una cuestión socialmente problematizada que logró ser escuchada y atendida estatalmente. Luego, participaron y tensionaron las formas para la atención de esta problemática, exigiendo y posicionándose como participantes del proceso de 
ejecución. Además, remarcamos que la institucionalización y formalización de estas políticas (mediante sanción de una Ley o Resolución de Directorio) no pueden pensarse sin un largo periodo “informal” en el que éstas fueron corporizándose.

En el PNNH, comienza a llevarse adelante en el año 2000, un comanejo con la constitución formal de la primera comunidad mapuche dentro de jurisdicción del parque, siendo institucionalizado por Resolución recién en abril de 2012, momento en el que se piensa su "inicio formal”. En este sentido, lo contingente, informal y no institucionalizado de estas políticas no puede definirse por oposición a lo establecido, formal e institucionalizado, sino que es en los intersticios de estas políticas donde pueden verse las reconfiguraciones de límites y posibilidades de acción política desde la agencia indígena. Esto expone cómo las relaciones personales, los vínculos, lo "no escrito" y lo "no regulado" son centrales para el desarrollo e implementación cotidiana de estas políticas, incluso, en contradicción con la propia definición de las mismas.

Desde este enfoque, el comanejo del PNL tiene su "inicio real" a fines de la década de 1980, cuando empieza a instalarse la cuestión indígena a nivel local. Cuando decimos "inicio real” nos referimos al inicio en la práctica, algo que antecede a la formalización de las políticas institucionales. La APN es una institución fuertemente burocrática, por lo que para que una política sea entendida como institucional se necesitan ciertos procedimientos. Por este motivo en ambos casos de comanejo el inicio real del mismo dista del inicio formal por casi diez años. En este proceso, lo que intentamos mostrar es que esta política es resultado del accionar conjunto de los mapuche y de distintos actores institucionales, por lo que sería un error limitar el análisis a si el comanejo surge de las políticas de la APN como respuesta a una demanda local. Como buscamos mostrar entendemos al comanejo como un proceso que no puede entenderse por fuera de la práctica situada y del hacer de aquellos involucrados. En 1989 se da la experiencia de veranada en Huaca Mamuil y se efectiviza la primera ley de propiedad comunitaria de la comunidad Curruhuinca, con la Ley 23.750/89 de 10.500 Ha; luego con la Ley 25.510/01 se realiza la transferencia a la comunidad Cayún. Estas leyes implican el reconocimiento de la propiedad comunitaria mapuche de grandes extensiones de las tierras más caras de la zona. Durante esta década, tienen lugar proyectos de desarrollo e infraestructura para mejorar la calidad de vida de las poblaciones, permitiendo el acceso a recursos, el aumento de la capacidad de acción e implementación de los mismos, así como una incipiente apertura de la participación. Resaltaremos que las experiencias de manejo compartido se dan en un marco de informalidad institucional, más relacionadas con el compromiso y las voluntades particulares de determinados trabajadores de parques (técnicos, administrativos y guardaparques), que a una política institucional de la APN.

En el PNNH el proceso fue inverso. En el 2000, cuando se realiza el Primer Taller de Territorio Indígena Protegido y se formaliza el comanejo del PNL, empieza a hablarse de comanejo en Nahuel Huapi referenciando a la relación con las comunidades reconocidas por la institución. A diferencia de Lanín, en esta primera etapa "informal" era la jerarquía administrativa (directorio, intendente, funcionarios) la que hablaba de comanejo, mientras que con los guardaparques y personal técnico vinculados a las comunidades en sus territorios existían tensiones. Así, mientras en el PNL el proceso fue gestándose "en terreno", en el PNNH durante los primeros años las relaciones se dieron en forma directa con el intendente o directorio, siendo decisiones tomadas a un nivel administrativo/jerárquico, siguiendo la línea política que empezaba en la APN y se formalizaba en el PNL. En ambos parques existieron visiones contingentes y no formales de los comanejos durante casi diez años en las que estas políticas se fueron desarrollando en los intersticios institucionales, en relaciones personales en las que las agencias de los sujetos involucrados devinieron fundamentales para su desarrollo. Sería impensable hoy el comanejo del PNNH sin tener en cuenta los vínculos directos entre referentes mapuche y el entonces intendente en los inicios del 2000 donde esta política no existía por fuera de esta relación. Lo mismo sucede en el caso del PNL en la relación particular de algunos técnicos y guardaparques con referentes comunitarios. Por eso hablamos de relaciones personales que moldean la política.

Asimismo, tanto en el PNNH como en el PNL el desarrollo de los comanejo está fuertemente relacionado a acciones mapuche por la defensa de los territorios comunitarios cuestionando lógicas institucionales y tensionando aquello que se entendía por "ser mapuche". En el PNNH este proceso fue aún más disruptivo debido a que -a diferencia del PNL- no fueron reconocidas comunidades indígenas dentro de su jurisdicción desde su creación en 1934.Así, durante el periodo “informal” del comanejo, la discusión para su implementación 
se centró en la autenticidad de las comunidades conformadas dentro de jurisdicción del parque, a partir de históricos pobladores con PPOP o de poblaciones “intrusas” que habían sido desalojadas. En este proceso, las propias comunidades fueron discutiendo y resignificando lo que se entendía por "ser mapuche" en el PNNH, llegando al momento de institucionalización de esta política con parte de estas discusiones (qué implica ser comunidad, quién es mapuche y quién no, cómo debe ser un mapuche dentro de un parque) ya saldadas, o al menos no como obstáculo para el desarrollo de la política.

En ambos casos, este proceso previo a la institucionalización y formalización de comanejos fue construyéndolos, y la(s) agencia(s) indígenas devinieron fundamentales para discutir su implementación, redefiniendo al sujeto indígena que podía ser parte de ellos. En este último caso, destacamos que las alianzas de conservación a nivel global se piensan con determinado tipo de indígenas, denominados como "indio verde" o "buen salvaje ecológico" (Dumoulin, 2005) que entran en tensión con las formas de "ser mapuche" en la cotidianeidad de las comunidades de estos parques.

De modo similar, el ReTeCI también da cuenta de un inicio informal. La Ley 26.160tiene su "inicio formal" en 2006, no obstante, su "inicio real" puede rastrearse con anterioridad en diferentes movilizaciones indígenas, instancias participativas, de discusión, y diseño que culminaron con su sanción. Dicha ley es una respuesta a demandas indígenas de larga data, quienes demandaban defensa, cese de atropellos, protección y reconocimiento de sus territorios. El relevamiento ordenado por esta ley cobra su forma final en 2007, luego de meses de trabajo conjunto entre técnicos del INAI y referentes indígenas, nucleados en los espacios de participación indígena reconocidos estatalmente. Respecto a su ejecución rionegrina, si bien comienza formalmente en junio de 2009, con anterioridad se realizaron reuniones zonales promovidas por referentes mapuche para difundir la ley, compartir información con las comunidades, y pensar estrategias en torno a quiénes serían alcanzados por el relevamiento, es decir quiénes serían los sujetos de esta política, y a cómo establecer prioridades sobre las primeras comunidades a relevar, qué información brindar y qué resguardar frente al ETO. Estas cuestiones tenían por objetivo crear criterios y formas de abordaje comunes, forjando alianzas entre comunidades y ser planteadas al ETO cuando iniciaran sus tareas, con el fin de ser los propios indígenas quienes marcaran el rumbo del relevamiento desde su inicio formal.

Entonces, más allá de las fechas que establecen el inicio de las políticas, éstas son el resultado o la materialización de procesos de larga duración que evidencian particulares formas de relacionamiento entre estado e indígenas. Formas que están moldeadas por las expresiones de múltiples agencias que cuestionan las lógicas que sustentan estas políticas y desafiaron las formas en las que se implementaron "formalmente".

\section{Agencia indígena en la definición del sujeto de reconocimiento}

Ambas políticas se aplican sobre "comunidades indígenas", es decir que el reconocimiento en tanto "indígenas" y los derechos diferenciales derivados de este reconocimiento están sujetos a la pertenencia a una “comunidad” jurídicamente reconocida por el estado, a través de su inscripción en el ReNaCI ${ }^{1}$. La categoría de "comunidad indígena”, en tanto concepto jurídico, adquirió una nueva acepción con la actualización de la cuestión indígena en la agenda pública nacional desde mediados de 1990. Actualmente, está vinculado al reconocimiento por parte de distintas instancias estatales de qué agrupación es o no una "comunidad indígena", a través de determinados pasos burocráticos. En el marco de políticas públicas se define como "comunidad indígena" a un grupo de familias que habita un territorio determinado (con límites claros e identificables) y que autoadscriben a un pueblo originario. A esto se agrega el supuesto de que el territorio debe haber sido habitado por ellos de manera ininterrumpida desde tiempos “ancestrales”. En las definiciones de ambas políticas no se tienen en cuenta los procesos y el accionar del estado sobre estos colectivos. Así, las políticas parten de entender alas comunidades como formaciones "naturales", propias de cualquier grupo indígena, que deben permanecer inmodificables a lo largo del tiempo (Trentini, 2017). Justamente, como mostramos en ambos casos, las demandas indígenas tensionan esta visión esencialista, y en consecuencia los requisitos exigidos para ser reconocidos como sujetos de derecho.

En relación a lo anterior, en la ejecución del ReTeCI inicialmente se convocó a comunidades con personería jurídica para confeccionar un "listado de comunidades a relevar", de aproximadamente 80 comunidades. A lo 
largo del proceso de relevamiento fue incrementándose el número de comunidades interesadas en acceder, debido a la divulgación y trascendencia de información. Así, otras comunidades peticionaron formalmente ante el INAI y CODECI, para ingresar al listado. El último listado tenía 126 comunidades. Incluso, en este proceso, se fueron tensionando los requisitos necesarios y excluyentes, como el estar inscriptos en algún registro, la demostración de pertenencia mapuche y los signos (válidos estatalmente) de ocupación territorial a georreferenciar. A raíz de esta "explosión de autoascripciones" (Guiñazu,2017) evidenciada por el aumento de comunidades solicitantes se profundizaron cuestionamientos en torno a su falsedad, inautenticidad u oportunismo.

Actualmente, la categoría "comunidad indígena” tiene implicancias específicas, ya que al ser reconocido mediante el otorgamiento de una personería jurídica, un grupo es legitimado por el estado como "diferente”, otorgándole acceso a derechos diferenciales. No obstante, en ambas políticas, esta categoría termina cristalizándose, invisibilizando procesos históricos particulares y ocultando históricas relaciones de poder y desigualdad. Destacaremos que el reconocimiento de una "comunidad indígena" por parte del estado trae aparejada una forma particular de vincular "la identidad”(entendida como sinónimo de cultura y definida por sumatoria de rasgos) y "el territorio"(entendido como un espacio factible de ser delimitado espacial y temporalmente) (Trentini, 2017). Sin embargo, durante la implementación de ambas se manifestó la imposibilidad de establecer relaciones fijas entre identidad cultural y territorio. Esto se reflejó en la práctica del ReTeCI en el proceso de construcción cartográfica, inicialmente guiada por tres supuestos tendientes a la búsqueda y encuentro de territorios comunitarios unificados, a su delimitación por una línea o frontera del resto del espacio, y a la búsqueda y marcación de gran cantidad de signos a georreferenciar, como demostración de que estaba "realmente ocupado" (Guiñazu,2017b). Estos supuestos evidenciaban una particular lógica de visualización estatal que buscaba relevar territorios ideales. No obstante, los mismos comenzaron a disputarse y tensionarse en la práctica a través de la articulación de estrategias entre técnicos e indígenas. Como corolario, se expuso la complejidad de los territorios indígenas, en lo referido a cuestiones de conflicto, despojo y violencia, así como también a sus diferentes "usos", los cuales difieren de aquellos que el estado, a través del relevamiento, buscaba.

En el comanejo del PNNH puede encontrarse esta tensión entre la definición de una "comunidad indígena” y la realidad vivida por las comunidades del parque. La conformación de comunidades mapuche en el PNNH a partir del 2000 cuestionó la idea de un parque "sin indios" en el que no habían existido históricamente comunidades reconocidas oficialmente. En este sentido, las comunidades cuestionaron los principios del "programa de la doble conservación" que sustentan las definiciones modelo de esta política, tensionando supuestos sobre su autenticidad/inautenticidad. La mayoría no encajaba en la definición ideal de una alianza estratégica de conservación, porque no podían comprobar haber convivido con la naturaleza desde tiempos inmemoriales sin destruirla, ya que habían sufrido un largo proceso de desalojos y relocalizaciones desde la creación del parque. Además, porque no mostraban signos de "indianidad" evidentes, factibles de catalogarse como tradicionales, que debían conservarse junto con la biodiversidad territorial. Por lo tanto, no entraban dentro de la lógica de las políticas esencialistas de reconocimiento de la APN, asociadas al "nativo ecológico" y a pautas concretas de cómo ser indígena. Asimismo, se fue generando una comparación con las comunidades del PNL, ponderándolas como "verdaderas" y como punto para medir la "autenticidad" de las comunidades del PNNH.

Al conformarse las comunidades del PNNH, no practicaban ceremonias, no hablaban mapuzungun (idioma mapuche), no usaban vestimentas tradicionales, es decir, no contaban con marcas diacríticas que manifestaran la "diferencia cultural", necesaria para implementar un comanejo. Pero, fundamentalmente, una cuestión las volvía inauténticas para la APN: no vivían en el territorio, lo cual dificultaba su marcación. Ante esta situación no se profundizaba sobre sus causas, ni sobre los desalojos y políticas de vaciamiento de los territorios mediante los PPOP como condicionantes de ocupaciones continuas dentro del parque. Para el caso de alianzas estratégicas de conservación, vivir en el territorio era también garantía de saber conservarlo. De esta manera, el problema radicaba en el vínculo construido entre algunas prácticas prohibidas por la APN y la calificación como "indios aculturados", "peligrosos" para la conservación. Nuevamente se realizaron comparaciones con el comanejo del PNL, remarcando que estas comunidades habían existido "siempre” en los territorios que se les reconocían.

Con todo, sostenemos que estas tensiones están basadas en las distintas formas existentes de "ser comunidad”. Formas que no encuentran lugar en las definiciones o regulaciones ni de las políticas analizadas. En 
ambos casos, desde sus letras, existe una sola forma posible de serlo, basada en fuertes estereotipos y esencialismos. Pero principalmente basada en la delimitación clara de un territorio y la permanencia histórica en el mismo en función de lo que se supone debe ser la relación entre conservación de la biodiversidad y diversidad cultural. Esta relación supone "usos tradicionales" sobre un territorio espacial y temporalmente definido y una cultura factible de ser diferenciada y observada a través de rasgos diacríticos, entendida como sinónimo de identidad.

\section{Agencia indígena en la (de)construcción de la(s) frontera(s) entre estado y sociedad civil}

Las políticas presentadas delimitan entre estado e indígenas -como parte de la sociedad civil- construyendo una frontera que en la práctica deviene borrosa. Este borramiento se vincula a múltiples expresiones y despliegues de agencia(s) indígena(s). Además, muestran que sus sujetos de derecho no son entendidos como meros beneficiarios, sino como actores clave del diseño y ejecución, mediante espacios de participación habilitados estatalmente y disputados desde la agencia indígena. Sostenemos que esta delimitación está en permanente cambio y que, al entenderla como opuesta, habilita asociaciones entre la cooptación y la participación intraestatal de algunos referentes indígenas. Durante la implementación de estas políticas reiteradamente se manifiesta que los indígenas se "burocratizaron", sin tener en cuenta sus procesos previos, mediante los que idearon modos de vincularse, dialogar y discutir con "el estado". Este pensamiento dicotómico entiende la participación desde una lógica binaria y rígida entre empoderamiento y cooptación, cuando en la práctica existen diversos tipos de participación que rompen y desarman la idea estado/sociedad civil.

La participación indígena en la gestión estatal, puede entenderse como reconstrucción hegemónica, como un intento de mejorar la gobernabilidad reduciendo la conflictividad, como mecanismo de expansión del control y dominación estatal de la población y el territorio. No obstante, puede analizarse como profundización de la democracia participativa en las estructuras estatales, a través de la implementación de esquemas de participación social a nivel local, que institucionalizan formas alternativas y multiculturales de democracia (Van Cott, 2004). Nuestra propuesta, comprende estas PPP como campos de disputa que posibilitan la ampliación de derechos ${ }^{2}$, rompiendo con la mirada dicotómica de cooptación/resistencia, gobernabilidad/emancipación, autonomía/heteronomía. Al pensar la relación estado/sociedad civil dicotómicamente se destaca al estado como el máximo garante de la construcción de la nación, y a la sociedad civil por su potencialidad para presionar "desde abajo”, frenando acciones estatales monolíticas y avasallantes. Frente a esto, cuestionamos la mencionada frontera, entendiéndola como un efecto de poder que permite pensar al estado entre (y no automáticamente distinto de) otras formas institucionales, a través de las cuales las relaciones sociales son vividas (Sharma y Gupta, 2006).

En este sentido, pensamos cómo el surgimiento de comanejos ha sido el conflicto, mientras los logros fueron resultado de acciones de protesta y movilización. En el PNL esto acontece conjuntamente entre comunidades y trabajadores, tensionando al estado con sus demandas y consiguiendo avances mediante la presión, generando una alianza y una confluencia en la participación en el diseño, formulación e implementación de la política. En el PNNH la metodología es la misma, aunque llevada adelante por las comunidades, generando tensiones con trabajadores con los que después implementan la política. Estas acciones marcan una brecha entre "los mapuche" y "el Parque". Aquellos miembros de la institución que acompañan y ponen el cuerpo cotidianamente viven estas instancias como un retroceso que mina la confianza y el trabajo compartido; mientras los mapuche no se sienten acompañados en su lucha por sus compañeros, asociando esto a limitaciones institucionales.

Como explicitamos, esto muestra una diferencia en cómo la política de comanejo se fue construyendo y en el accionar indígena en cada caso. En el PNL, previamente a la institucionalización se desarrollaron experiencias concretas y locales de administración y control territorial, construyendo confianzas, militancias y confluencias entre referentes e integrantes de las comunidades y los trabajadores de parques, articulando acciones directas y modificando las relaciones históricas de parques con las comunidades. En el PNNH el comanejo se inició por una articulación superestructural, lejos del nivel local que caracterizó la experiencia de Lanín.

En el PNNH la dicotomía entre Parques (como sinónimo de estado) y comunidades se profundiza en reuniones periódicas que "teatralizan" esta frontera, donde cada sector define y defiende intereses y expectati- 
vas tensionadas con las del otro. Sin embargo, las expectativas sobre esta PPP no se dividen entre Parques/ mapuche, sino en función del lugar que cada uno ocupa: para los referentes de las comunidades la prioridad es resolver cuestiones inmediatas (construir casas, puentes, mejorar los caminos), mientras que para los técnicos, funcionarios y directivos de la APN y para los dirigentes indígenas prima una visión a largo plazo de lo que construir un comanejo implica. Este no es un dato menor, en tanto los dirigentes indígenas son quienes participaron de discusiones globales sobre conservación y participaron activamente del proceso de construcción del comanejo en el PNL. Así, desde la institución se diferencia entre los que "entienden” y "saben” lo que es el comanejo, y los referentes comunitarios que lo ponen en "peligro" con sus reclamos.

Asimismo, esta supuesta división entre comunidades/parques también se tensiona al pensar la situación en la que se encuentran los trabajadores del parque que además son parte del Pueblo Mapuche. En la dinámica cotidiana, salvo que se encuentren trabajando por elección de las comunidades, son considerados como "gente de Parques", sin embargo, en momentos conflictivos, en los que se debe definir si "juega" para Parques o las comunidades, se espera la explicitación de su posicionamiento, generando discusiones donde uno y otro lado consideran que su lealtad debiera ser con "los suyos". Por ejemplo, el apoyar situaciones de toma o corte de caminos parece no ser lo que se espera de un trabajador del PNNH, y no apoyarlo lo tensiona como parte del Pueblo Mapuche.

En el PNL, las estrategias desplegadas por los actores buscan dispositivos para avanzar y mejorar posibilidades, reconfigurados en el transcurso del proceso, siendo por momentos más colectivas o individualizadas, más de base y locales o jerárquicas y nacionales. Una primera etapa destaca el protagonismo de dirigentes (werkenes) de la Confederación Mapuche de Neuquén y un proceso incipiente de fortalecimiento de las comunidades y sus autoridades. Además, se caracteriza por el dinamismo, periodicidad y participación en las reuniones del Comité de Gestión; mientras que a los Comités locales les cuesta comenzar a funcionar y que sus integrantes y autoridades comunitarias se lo apropien y participen. Una segunda etapa, descentraliza la organización mapuche al interior (creación de las zonales) y el PNL (Comités locales y Unidad de Gestión Descentralizada) y el Comité de Gestión pierde relevancia (sus reuniones pierden periodicidad hasta dejar de realizarse), adquiriendo protagonismo los Comités Locales. Al inicio, el Comité de Gestión fue el espacio dinámico, pero años después deja de funcionar y el comanejo subsiste a nivel local. Así, se pone en ejercicio la autoridad mapuche comunitaria, tomando decisiones en relación a las veranadas, recolección de piñones y leña, etc., que hacen que -a nivel territorial- las comunidades se apropien del comanejo.

Por su parte, el ReTeCI evidencia una “doble relectura del concepto de participación” (Guiñazu 2017, p.162). La primera relectura trastoca los supuestos de despolitización de la participación y del compromiso de quienes participan, mientras quela segunda trastoca los supuestos de necesaria separación de la sociedad civil del estado y sus estructuras. Esta segunda relectura expone que se han modificado las formas de participación, entendiéndose a la relación entre indígenas y estado como una "participación intraestatal" (Guiñazu, 2017, p.164) que sucede en las mismas instituciones del estado y con financiamiento estatal, no sólo para las actividades de ejecución de la política, sino también para pago de salarios de estos participantes. Este tipo de participación tensionó hacia el interior del movimiento indígena, existiendo múltiples posicionamientos respecto a lo que entienden como "ser parte del estado" o no. Entre estos destacan argumentos en torno a que los espacios estatales deben ser entendidos como herramientas disponibles y ocupados en ese sentido, para asegurarse de utilizarlas en su máximo beneficio y potencial. Mientras que otros argumentan que no se puede participar de políticas duramente criticadas por sus limitaciones A partir de lo anterior se entiende que algunas comunidades o miembros de comunidades deciden participar mientras que otras no, y que esta decisión de participar no implica cooptación. Asimismo, remarcamos que la agencia debe entenderse y analizarse trascendiendo dicotomías como dominación o resistencia, dando lugar a sus múltiples expresiones.

Con lo expuesto mostramos que tratándose de políticas públicas que se abren a la participación indígena, atendiendo a las normativas vigentes que así lo ordenan, evidencian en su proceso de ejecución efectos tanto de esta participación como de la agencia indígena. Una participación y agencia que trasciende las dicotomías de aceptación/rechazo o de empoderamiento/cooptación, mostrando variados despliegues de acciones posibles y disputadas desde el "interior” mismo de estas políticas. Esta trascendencia se materializó en efectos concretos, como los desafíos y tensiones respecto de liderazgos y representaciones indígenas, las cuales no se agotan en 
los organismos reconocidos o validados estatalmente para el diálogo con el estado. También, en estrategias, alianzas y articulaciones entre la heterogeneidad de actores intervinientes y entre indígenas y técnicos en particular, fortaleciendo acciones y disputas a los límites y alcances establecidos. Igualmente, en las reformulaciones y cambios sugeridos y plasmados en los modos de llevar a cabo el programa de relevamiento, producto de exigencias, tomas de posición y disputas al estado nacional, representado en esta instancia por el INAI, y en disputas, tensiones y modificaciones por las maneras de implementar un comanejo, discutiendo con la visión estatal, representada por la APN.

El problema, al momento de pensar en PPP es separar a las comunidades indígenas del estado, construyéndolas como exteriores y/o en contra del estado (Li, 2002). Según Li el problema surge cuando se intenta convertir esta imagen simplificada sobre las comunidades en una realidad, entendiéndola como la base de la estrategia en la búsqueda de derechos y justicia. Siguiendo su planteo, los comanejos y el relevamiento son útiles como estrategia en esta disputa para quienes representan la imagen ideal, pero esto raramente sucede en las configuraciones actuales de estos grupos, y por lo tanto, estas políticas imponen limitaciones, condicionando los derechos a visiones esencialistas inaplicables. Frente a esto, hemos dado cuenta de cómo la(s) agencia(s) indígena(s) actúan disputando estas limitaciones para lograr una ampliación de sus derechos.

\section{Conclusiones}

En los últimos años, desde el estado nacional se ha manifestado el interés de interactuar con los indígenas y fomentar su participación en políticas públicas que les competen, creando diferentes instancias estatalmente legítimas. De esta manera se han regulado y normalizado modos y canales de interacción y participación. No obstante, la aceptación de participación por parte de los indígenas no implica pasividad y sumisión, sino múltiples formas de ejercicio de su agencia política. En este sentido, la necesidad de instaurar una política definida previamente "choca” con la idea de agencia que construye y reconstruye estas políticas en la práctica.

Analizar estas políticas en sus prácticas da cuenta de distintas formas de participación y de exclusión, de relaciones contradictorias, de heterogeneidades institucionales y de avances y retrocesos permanentes. Esta visión expone los límites y condicionamientos, pero también las posibilidades de agencia que los desafían, negocian y disputan, reconfigurando la propia forma de "hacer la política". El problema radica en pensar a los comanejos y al relevamiento como "algo dado", invisibilizando las prefiguraciones que condicionan su forma de aplicación. Esas prefiguraciones generan una escala de aplicación de la política desde la que se construyen ideas de éxito y fracaso, de funcionamiento y no funcionamiento y desde las que se sostiene que hay quienes entienden y quienes no entienden la política.

En este sentido, es necesario dejar de lado las definiciones técnicas o la letra escrita de estas políticas y atender a los procesos que intervienen en su construcción e implementación en contextos situados marcados por una historia particular y por relaciones de poder desiguales. Las PPP e indigenistas son producto de esa historia y de esas relaciones, son arenas de disputa por la que los involucrados transitan y en las que su capacidad de agencia permite reformular lógicas impuestas por modelos globales o experiencias previas, generando posibilidades de acción política que repercuten en un mejoramiento de la calidad de vida y en una ampliación de derechos. Tanto los comanejos como el relevamiento implican nuevas posibilidades para la disputa por territorios, recursos y derechos, y por lo tanto no son políticas que puedan ser analizadas desde una perspectiva netamente evaluativa, que concluya en que "fracasaron" o "no funcionaron", porque esta evaluación se sustenta en lo que las definiciones de estas políticas sostienen, invisibilizando las múltiples agencias indígenas.

El análisis de estas PPP invita a repensar "lo indígena" en ellas establecido, por no encajar con las realidades de las comunidades. Lejos de definirlo por rasgos distintivos y en relación a un territorio específico entendemos que "lo indígena" debe pensarse como formas de agencia y politicidad (Briones, 2013) que entran en tensión con las políticas de reconocimiento estatal que definen una forma de "ser diferente" y en consecuencia la forma habilitada/permitida para poder participar de estas políticas. Frente a esto entendemos que son las formas en las que se disputa, se negocia, se resiste y "se hace la política" las que permiten ir más allá de "la política” para dar cuenta de formas situadas de comanejar, de relevar y de "ser indígena”. 


\title{
Notas
}

\begin{abstract}
${ }^{1}$ Requisitos de inscripción: nombre y ubicación geográfica, reseña histórica que acredite origen étnico, pautas de organización, nómina de integrantes y parentesco y mecanismos de inclusión/exclusión de miembros (INAI, 2015).

${ }^{2}$ Tal como adelantamos, esta ampliación de derechos, se materializa en el caso del relevamiento territorial en la disputa de tiempos de ejecución, de presupuesto asignado, de cantidad de comunidades a relevar, de conceptos utilizados - “territorio", "tradicional”, "actual”, "público", entre otros- y de efectos y alcances de la política pública. Para el caso del comanejo, se ha ampliado el alcance de la política al tensionar las nociones de "indígena” y "comunidad”, posibilitando que mas comunidades fueran reconocidas como sujetos de comanejo.
\end{abstract}

\section{Bibliografía}

Abers, R. N. (2007). Organizing for governance: building collaboration in Brazilian river basins. World Development, 35(8), 1450-1463.

Abers, R., \& Keck, M. E. (2009). Mobilizing the state: The erratic partner in Brazil's participatory water policy. Politics \& Society, 37(2), 289-314.

Ameghino, N. (2011). ¿Interculturalidad hegemónica o emancipatoria?: Una aproximación a la gestión intercultural en Neuquén, Argentina. Cultura y representaciones sociales, 6(11), 83-108.

Ameghino, N. (2013). Un pueblo, dos Estados: participación mapuche en el Estado. Si somos americanos, 13(1), 171-197.

Amend, Stephan y Amend, Thora (eds). (1991) ¿Espacios sin habitantes? Parques Nacionales de América del Sur. UICN. Caracas: Editorial Nueva Sociedad.

APN.(2001). Plan de Gestión Institucional para los Parques Nacionales.

Borrini, G. (1997). Manejo participativo de Áreas Protegidas: adaptando el método al contexto. Ecuador, Quito: UICN-SUR.

Briones, C. (2005). Cartografías argentinas: políticas indigenistas y formaciones provinciales de alteridad. EA.

Briones, C. (2007). Reconocimientos y políticas respecto del derecho de los Pueblos Indiìgenas a sus tierras/ territorios en Argentina: un balance. En: Ramos, A. (ed.) Constituic?oPesnacionais e povosindiigenas. Brasilia, Brasil.

Briones, C. (2013). Formas de enraizarse en la mapu: procesos de formación de comunidad en Norpatagonia, Argentina. VII Congreso Internacional CEISAL, Oporto.

Carenzo, S. y Trentini, F.(2013). Producción de territorialidades indígenas y gestión de áreas protegidas: el paradigma de la doble conservación en la Argentina. En Balazote y Radovich (comps.) Estudios de antropología rural. Buenos Aires, Argentina: Editorial de la FfyL.

Carpinetti, B. (2005). De la expulsión al comanejo. APN.

Dumoulin, D. (2005). ¿Quién construye la aureola verde del indio global? El papel de los distintos actores transnacionales y la desconexión mexicana. Foro Internacional XL (1) 35-64.

Guiñazú, S. (2017). La performatividad de las políticas públicas: modalidades de interacción e interpelación entre Estado, sociedad e indígenas en el proceso de ejecución de una política pública indigenista, 2006-2017. Revista Estado y Políticas Públicas (9)145-167. 
Guiñazú, S. (2017b). La producción de mapas sobre las territorialidades indígenas en el marco del Relevamiento Territorial de Comunidades Indígenas en Río Negro. Revista TEFROS, 15(1), 153-187.

Guiñazú, S. (2018). El interjuego entre la normalización estatal y agencia indígena en la ejecución del relevamiento territorial de comunidades indígenas en Río Negro, Argentina. Antropologías del Sur, 5(9), 173-197.

Grossberg, L. (2014). We gotta get out of this place: Popular conservatism and postmodern culture. Routledge.

Li, T. (2002). "Engaging Simplifications: Community-Based Resource Management, Market Processes and State Agendas in Upland Southeast Asia”. World Development 30(2) 265-283.

Notzke, C. (1994). Aboriginal people and natural resources in Canada.Ottawa, Canadá: CaptusPress.

Ortner, S. (2016). Antropología y teoría social: cultura, poder y agencia.Buenos Aires, Argentina: UNS

Ramos, A. y Delrio, W. (2005). Trayectorias de oposición. Los mapuches y tehuelches frente a la hegemonía en Chubut. Cartografías Argentinas. Políticas indigenistas y formaciones provinciales de alteridad, 73-108.

Sharma, A. y Gupta, A. (2006). The antropology of the state: a reader. Oxford: Blackwell Publishing

Shore, C. (2010). La antropología y el estudio de la política pública: reflexiones sobre la "formulación” de las políticas. Antípoda. Revista de antropología y arqueología, (10), 21-49.

Stevens, S. (1997). ConservationThrough Cultural Survival. Indigenous Peoples and Protected Areas, Washington, United States: Island Press.

Trentini, F. (2014). Pueblos indígenas y áreas protegidas: procesos de construcción de identidades y territorialidades en el co-manejo del Parque Nacional Nahuel Huapi. Tesis de Doctorado, FFyL, UBA.

Trentini, F. (2017)“Os comanejo de áreas protegidas na Argentina como formas de juridicidade em disputa”. En: Verdum, R. y Ioris, E. (Org.) Autodeterminação, autonomia territorial e acesso à justiça: povos indígenas em movimento na América Latina. Río de Janeiro: ABA, 71-98.

Van Cott, D. L. (2004). Los movimientos indígenas y sus logros: la representación y el reconocimiento jurídico en los Andes. América latina hoy, (36). 